\title{
»Soziale Ungleichheit - Kulturelle Unterschiede« Anmerkungen zum 32. Kongress der Deutschen Gesellschaft für Soziologie
}

\section{Von Helmuth Berking}

Traut man dem Bild, das sich aus den Kommentaren der Medien zusammenfügen lässt, waren es vor allem zwei Leitmotive, die den 32. Soziologiekongress, der vom 4.-8. Oktober 2004 an der Maximilian-Ludwigs-Universität München stattfand, beherrschten: die durch die »Ökonomie der Aufmerksamkeit« forcierte zeitaktuelle Problematik der sozialen Ungleichheit sowie jene bereits auf dem Geburtsschein der Soziologie vermerkte Dauerkrise des Faches. Die Berichterstattung gewinnt ihren distinkten Charakter schließlich über eine geographische Imagination, die sich nicht nur in sprachlichen Formeln wie die »deutsche Sozialwissenschaft «, sondern vielmehr noch darin Ausdruck und Geltung verschafft, dass die journalistischen Relevanzmuster offenkundig nur das als berichtenswert identifizieren, was die deutsche Sozialwissenschaft über die deutsche Gesellschaft erforscht. Die zugegebenermaßen grobe Formel könnte deshalb lauten: Der 32. Kongress der deutschen Gesellschaft für Soziologie erscheint in seiner medialen Präsentation als eine landeskundliche Veranstaltung, und die gesellschaftliche Wertschätzung der Soziologie bemisst sich an diesen, ihren landeskundlichen Kompetenzen. Nicht die Selbstbeobachtung der Gesellschaft, die manch kühner Geist längst zur Weltgesellschaft hat werden lassen, sondern die Beobachtung der eigenen nationalstaatlichen Gesellschaft liefert die unaufgeklärte normative Rahmung für die Relevanzen und Wahrnehmungsmuster einer interessierten Öffentlichkeit. Dieser Befund ist umso erstaunlicher, als Globalisierungsrhetoriken, Transnationalismusdiskurse und Entteritorialisierungsvorstellungen in die Feuilletons ebenso wie in das ganz gemeine Alltagsleben längst Einzug gehalten haben.

\section{Heimspiel}

Die Diskrepanz zwischen Außenwahrnehmung und Binnenperspektive, zwischen journalistischem und akademisch- soziologischem Diskurs über den und auf dem Soziologiekongress verweist auf interessante Beziehungsprobleme. Soviel jedenfalls lässt sich belegen. Erstens: »Soziale Ungleichheit« und die »Krise der Soziologie« waren in der Tat zentrale Themen des Kongresses, während »kulturelle Unterschiede«, die andere Hälfte des Kongressthemas, die ja ihrerseits ein bezeichnendes Licht auf die gesellschaftlichen Semantiken der sozialen Ungleichheit zu werfen versprach, in der Soziologie wie in der Presse eher beschwiegen als analytisch fruchtbar gemacht wurde. Zweitens: der lokalspezifische Provinzialismus der Berichterstattung scheint auf irritierende Weise mit einer Art thematischem Provinzialismus von Teilen der akademischen Soziologie zu korrespondieren. Diese Wahlverwandtschaft findet im »methodologischen Nationalismus « - der unausgesprochenen aber wirkungsmächtigen Hintergrundannahme, den (eigenen) territorialen Nationalstaat als die exklusive Organisationsform des Sozialen zu setzten - ihr fundamentum in re. Drittens aber, und hier trennen sich die Wege: Die kritische Auseinandersetzung mit den kulturspezifischen Grenzen soziologischer Konzeptbegriffe, mit Eurozentrismus, Moderne und Modernisierung, Zivilgesellschaft und Staat etc. lieferte im Verein mit empirisch informierten Untersuchungen zu den dramatischen Folgen der globalen Diffusion von Wissensbeständen, Organisationsformen, institutionellen Arrangements einen weiteren zentralen thematischen Bezugsrahmen, dem in fast allen Veranstaltungen Rechnung getragen wurde. 
Die geographische Reichweite eines Soziologentages in Deutschland war wohl niemals größer als heute. Der interessierte Beobachter konnte durch fremde Welten wie durch Museen spazieren, von den Goldgräbern West-Afrikas (T. Grätz), durch die neuen globalen Mobilitätsräume (J. Urry) bis in die prekären Lebensverhältnisse der Megacity Bombay (S. Patel) oder einer Plattenbausiedlung in Budapest (A. v.d. Hagen-Demsky).

Natürlich lassen sich regionale Verdichtungen identifizieren. Aus historisch institutionellen Gründen und angesichts der radikalen wohlfahrtsstaatlichen Reformprozesse in der Bundesrepublik scheint die thematische Übergewichtung dieses geographischen Raumes selbstevident. Ob Hartz IV, die Gesundheits- und Bildungsreformen, oder der Beitritt der Türkei zur EU, der tagespolitische Informationsbedarf traf sich mit profunden soziologischen Analysen hinsichtlich aller gesellschaftlichen Funktionsbereiche. Dass die »unsichtbare Klassengesellschaft«(K.-S. Rehberg), zumindest in ihrer bundesrepublikanischen Version, in den Horizont der Wahrnehmung zurückgeholt, dass die Produktionslogiken der Unsichtbarkeit auf dem Arbeitsmarkt, in den Bildungsinstitutionen (M. Vester), aber auch im öffentlichen Diskurs (U. Volkmann; Ch. Lahusen) analytisch aufgeschlossen wurden, verweist ebenso auf ein gesteigertes Maß sozialwissenschaftlicher Selbstreflexion, wie die durchgängige Erkenntnis, dass und in welchem Ausmaß Ungleichheitsdiskurse nationalspezifisch geprägt sind. Schließlich wird hier unter substantieller Beteiligung der Sozialwissenschaften, über legitime Mitgliedschaft und entitlements, über institutionsspezifische Normalitätsunterstellungen und Standards der materiellen Lebensführung verhandelt.

\section{Soziologie und Europa}

Soziologie freilich ist und zeigte sich auf dem Kongress als weitaus mehr als die (eigene) Gesellschaft in Gedanken gefasst. Die Relativierung des »nationalen Blicks« (U. Beck), dessen ungebrochene Dominanz durch die mediale Repräsentation des Soziologiekongresses sich ja auf überraschende Weise empirisch noch einmal zu bestätigen schien, bezog sich im wesentlichen auf eine zweite, erweitere Geographie, die thematisch West- und Osteuropa umschloss, in ihrem Kern aber auf die Europäisierung zielte. Das Projekt Europa, die europäische Gemeinschaft als Vergesellschaftungsmodus und Institutionengefüge stellt eine dreifache Herausforderung für die Soziologie dar. Sie motiviert erstens nationalstaatliche Vergleiche etwa zwischen Klassen- und Sozialstrukturparadigmata als Interpretationen nationaler Problemlagen (M. Haller), die die nationalkulturellen Variationen soziologischer Ungleichheitstheorien anschaulich vor Augen führen und so die drängende Frage auf die Tagesordnung gesetzt haben, inwieweit soziologische Wissensbestände und Theorien aller Abstraktionsleistung zum Trotz, nicht doch lokal kontextualisierte Wissensbestände sind, die eingeführten Kategorien der deutschen Sozialstrukturanalyse zum Beispiel möglicherweise kaum hinreichen, um die spanische oder finnische Gesellschaft auch nur annähernd zu beschreiben.

Die Europäisierung fordert zweitens dazu heraus, das grundbegriffliche Inventar und das Theoriedesign der Soziologie, soweit es auf dem »methodologischen Nationalismus« aufliegt, zu revidieren. Die »Europablindheit der Soziologie« (U. Beck) lässt sich durch nationalstaatliche Vergleiche nicht überwinden. Die Formel »Regieren im Mehr-Ebenen-System«, mittels derer die Politikwissenschaft sich als relevanter Diskurspartner der Europäisierung etablieren konnte, reicht für eine Soziologie Europas nicht hin. Wie sich eine sozialräumliche Organisation sozialer Beziehungen beschreiben lässt, die zwar aus nationalstaatlich organisierten Gesellschaften besteht, ohne jedoch den Modi nationalstaatlicher Souveränität, politischer Partizipation und nationalkultureller Integration zu gehorchen, ist und bleibt bis auf weiteres eine theoretisch ebenso brisante wie empirisch unaufgeklärte Problematik. Die Theorie der reflexiven Modernisierung (Beck/Bonß/Lau) hat sich zumindest in dieser Hinsicht erfolgreich auf den Weg gemacht, den methodologischen Nationalismus zu 
verabschieden und die in sich widersprüchliche Dynamik grenzüberschreitender sozio-ökonomische und kultureller Prozesse analytisch zu erschließen.

Die dritte Herausforderung schließlich betrifft die Frage nach dem relationalen Charakter des Geltungsanspruchs soziologischer Konzeptbegriffe. Universalitätsansprüche, bescheidener: Generalisierungen sind das Markenzeichen wissenschaftlicher Diskurse. In dem gleichen Maße aber, wie die soziologischen Wissensbestände der ersten, nationalstaatlich fixierten Geographie durch die erweitere Geographie Europas relativiert wurden, wird nun auch der Geltungsanspruch europäischer und/oder westlicher Konzeptbegriffe durch eine dritte, eben globale Geographie in Frage gestellt und auf seine regionale Dimension hin zu begrenzen versucht. »Kulturelle Vielfalt« übersetzt sich so in die grundlagentheoretische Aufforderung, Soziologie nicht nur im Plural, sondern als distinkte Unternehmungen in spezifischen geographischen Räumen auszubuchstabieren.

\section{Die Welt der Moderne(n)}

In diesem Kontext gehörte die durch den Vorstand der DGS initiierte Einladung Indiens als Gastland, resp. die Teilnahme indischer SozialwissenschaftlerInnen als Repräsentanten und soziologische ExpertInnen einer »nicht westlichen Gesellschaft« zweifellos zu den an- und aufregendsten Ereignissen des Münchener Soziologiekongresses. Was wissen wir über die »größte Demokratie der Welt« jenseits der typischen Binarismen von »Moderne und Tradition«, von »the West and the rest«? Worauf gründet der Universalismus einer Soziologie, die ihre Konzeptbegriffe aus den regional begrenzten Erfahrungen jener »modernen westlichen Gesellschaften « entwickelt, deren Selbstthematisierung - paradox genug - auf historischer Einzigartigkeit fußt? Welche Geschichten lassen sich gegenüber der hegemonialen Position des Westens und seiner kategorialen Konstruktion der Welt »from the inside out«, eben als geographische Expansion Europas, in Stellung bringen? Ist das dominante Prinzip klassischer Modernisierungstheorien, geographische Distanzen in historische Abstände, die Kopräsenz des »Hier und Jetzt « in ein entwicklungsgeschichtliches »Nacheinander« zu übersetzen, nicht weiterhin, und aller Kritik zum Trotz, eines der wirkungsmächtigsten Motive westlicher Weltbildstrukturen?

Die Erwartung freilich, dass diese Fragen die gesteigerte Aufmerksamkeit des Publikums finden würde, wurde nur teilweise erfüllt. Der Dialog der (Wissens-) Kulturen stieß schnell an die Grenzen begrenzten Interesses. Während das Plenum: »Wege der Moderne und Formen der Zivilgesellschaft in Indien« noch eine durchschnittliche Zuhörerschaft fand, die sich mit den enormen Bedeutungsverschiebungen und Neuschöpfungen solch (westlich) vertrauter Konzepte wie Säkularisierung (R. Bhargava), Zivilgesellschaft (A.K. Giri) und Moderne (S. Kaviraj) konfrontiert sah, blieben die indischen Gäste auf der Abendveranstaltung über »Kaste« als zentrales Strukturierungsprinzip der modernen indischen Gesellschaft weitgehend unter sich. Die Abwesenheit des Dialogpartners ist umso erstaunlicher, als »Kaste « eine jener Fokussierungsmetaphern darstellt, mittels derer der Westen der nicht-westlichen Gesellschaft Indien den Status einer »traditionalen Gesellschaft« verleiht. Dabei hätte man lernen können, dass und wie tief der gesellschaftliche Diskurs über Kaste durch den britischen Kolonialismus strukturiert wurde, dass und in welchem Maße Kasten und Demokratisierung verwoben (D. L. Seth), Klassendifferenzierungen durch Kasten überlagert und Gerechtigkeitsvorstellungen im gegenwärtigen postkolonialen Indien mit Kollektivrechten verbunden und dementsprechend durch »caste politics« artikuliert werden (S. Kaviraj). Die Erfindung des Hindu-Nationalismus ist das (moderne) Resultat von demokratischer Modernisierung und Kastenpolitik.

Wie wenig das (westliche) Verständnis von Kaste als traditioneller Vergemeinschaftungsform mit dem postkolonialen state of the art zu tun hat, veranschaulichen jene Debatten um 
das Verhältnis von Staat und Zivilgesellschaft, in denen Kaste nicht als traditionales Residuum, sondern im Gegenteil als wesentliches Organisationsprinzip der Zivilgesellschaft thematisiert wird. »Civil Society«, zunächst, ähnlich wie »Hinduismus«, ein weiterer Import des Kolonialismus, markiert im heutigen Indien anders als in westlicher Kontextualisierung nicht die Sphäre der freiwilligen Selbstorganisation autonomer Individuen, sondern vielmehr die Revitalisierung kollektiver Akteure angesichts des Versagens des postkolonialen Staates. Dass der indische Staat eine Vielzahl »traditioneller« rechtsetzender Instanzen und Institutionen anerkennt, unter denen die »Kastenräte« nur eine, wenn auch wichtige Form, darstellen (S. Randeria), verdeutlicht einmal mehr den Abstand, der sich zwischen die Welten der zivilgesellschaftlichen Begrifflichkeit zu schieben vermag. »Rechtspluralismus«, die Kasten als autonome, gesellschaftlich selbstorganisierte Gruppen, das spezifische Verhältnis von Zivilgesellschaft und Staat, das Zusammenspiel von Religion, Ethnisierung und Demokratie das sind einige der zentralen Topoi einer multi-pluralen, nicht-westlichen, und dennoch »modernen« Gesellschaft.

Die Kontroversen um die Analyse der postkolonialen indischen Gesellschaft - einen instruktiven Überblick bietet der neue von Martin Fuchs und Shalini Randeria herausgegebene Sonderband der Sozialen Welt: »Konfigurationen der Moderne: Diskurse zu Indien« schnurren auf die Frage: Welche Moderne? zusammen. Für Indien gehört die koloniale Erfahrung konstitutiv zur Moderne. Allerdings reicht es völlig aus, daran zu erinnern, dass die Kategorie der »Kolonie« noch vor 50 Jahren ein ganz selbstverständlicher Begriff des politischen Diskurses in Europa war, um wahrnehmen zu können, dass es sich hierbei um eine geteilte, eine gemeinsame Geschichte machtpolitisch asymmetrischer, gleichwohl wechselseitiger Abhängigkeiten handelt. »The trouble with the English « heißt es in den >Satanischen Versen $<$, »is, that they don't understand their own history, because most of it happened overseas, so they don't know, what it means«.

\section{Geteilte Geschichte}

Von geteilter Geschichte sprechen, heißt freilich auch - und auch hierfür bietet das postkoloniale Indien hinreichend Anschauungsmaterial - die naive Vorstellung von der globalen Diffusion der europäischen Moderne als einen Prozess der Imitation, Anpassung und schlichten Übernahme ad acta zu legen. Das Moderne der modernen, nicht westlichen Gesellschaften lässt sich nicht über ein Mehr oder Weniger an Nachahmung westlicher Ideen und Institutionen, aber auch nicht ohne Rekurs auf diese Ideen und Institutionen erfassen. Dass Modernisierung nicht in Westernisierung aufgeht, sondern auf verschlungenen, soziologisch aufzuklärenden Wegen, nicht-westliche Gesellschaften dazu anstiftet, eigene kreative Antworten auf die Herausforderungen der Moderne zu finden, ist ja die Leitidee jenes Konzepts der »multiple modernity«, das Shmuel Eisenstadt in einem brillanten Vortrag anlässlich des 100 jährigen Jubiläums von Max Webers >Objektivitätsaufsatz $<$ und der >Protestantischen Ethik « noch einmal vorführte. »Multiple Modernities « war auch das selbstgewählte Thema der Sektion: Soziologische Theorien. Wer mit den instruktiven Vorträgen und Diskussionen der indischen Gäste im Kopf in diese Veranstaltung kam, erlebt ein interessantes deja vu. Denn hier wurde auf theoretisch höchstem Niveau die (europäische) Moderne noch einmal in Szene gesetzt und in ihrem einzigartigen Universalitätsanspruch bedingungslos verteidigt. Die spannenden Kontroversen, die sich aus der Konfrontation mit den indischen Konzepten zwangsläufig hätten ergeben können, fanden so allenfalls als imaginierte in den Köpfen des Publikums statt.

Ganz anders dagegen jene Veranstaltungen, in denen ReferentInnen aus unterschiedlichen Welt-Regionen zur Sache und Differenzerfahrungen zur Sprache kamen. Das Plenum über »Megastädte und europäische Städte« mag als gelungenes Beispiel für das erkenntnisauf- 
schließende Potential des Dialogs zwischen regionalspezifischen Wissenskulturen stehen. Was es für unsere Vorstellung von Urbanität bedeutet, wenn in einer Stadt wie Bombay 55\% der Einwohner in shanty towns auf $6 \%$ des städtischen Raumes leben, die Verfügung über Raum der entscheidende Marker für soziale Ungleichheit ist und die kulturelle Entwicklung der Stadt sich als komplizierte Geschichte vom »colonial cosmopolitanism« zum »Hindu-nationalism« erzählen lässt (S. Patel) ist eine ebenso offene Frage, wie die über Größe und Dichte als strukturierende Merkmale von Megacities evozierte Problematik von Integration und governance, für die die Soziologie an der Schwelle zum urbanen Zeitalter (R. Korff) kaum überzeugende Konzepte besitzt. Und - dass der global talk die Zukunft des Urbanen an die Megacities zu binden versucht, erwies sich im direkten Vergleich mit der europäischen Stadtentwicklung, die einen differenten Typus des Urbanen zu repräsentieren scheint, als eine interessierte Fiktion (P. Le Galès). In dieser thematischen und regionalspezifischen Verschränkung globaler Problemlagen traten die (Un-) Vereinbarkeiten und Grenzen sozialwissenschaftlicher Konzeptbegriffe überraschend deutlich hervor. Ob das insgesamt aber eher begrenzte Interesse an den soziologischen Reflexionen einer außereuropäischen Gesellschaft tatsächlich mit einer Art »kognitiven Provinzialismus « einhergeht, wie ein Kommentator kritisch anmerkte, mag dahingestellt bleiben. Die für die deutsche Universität charakteristische Departementalisierung von Soziologie und Sozialanthropologie/Ethnologie jedenfalls trägt sicherlich nicht zur notwendigen Horizonterweiterung bei.

Eine Soziologie, die sich weder auf ihre regionalen Wissensbestände verlässt, noch sich reflexionsarm dem >global babble< überlässt, hätte sich der dreifachen Herausforderung einer relationalen Verortung von Erfahrungsgehalt, Theoriegestalt und Geltungsanspruch zu stellen. Der 32. Soziologiekongress der Deutschen Gesellschaft für Soziologie hat in dieser Hinsicht eine wichtige Wegmarke gesetzt.

Prof. Dr. Helmuth Berking TU Darmstadt, Inst. f. Soziologie Residenzschloss, 64283 Darmstadt berking@ifs.tu-darmstadt.de 